\title{
Dermatology
}

\section{Reflectance Confocal Microscopy for Noninvasive Monitoring of Therapy and Detection of Subclinical Actinic Keratoses}

\author{
M. Ulrich \\ D. Krueger-Corcoran \\ J. Roewert-Huber \\ W. Sterry \\ E. Stockfleth
}

S. Astner

Skin Cancer Center Charité, Department of Dermatology, Charité University Medicine, Berlin, Germany

\section{Key Words}

Actinic keratoses $\cdot$ Noninvasive diagnosis $\cdot$ Imiquimod $\cdot$ Monitoring $\cdot$ Confocal microscopy

\begin{abstract}
Background: Actinic keratoses (AK) represent cutaneous carcinoma in situ and have previously been evaluated by reflectance confocal microscopy (RCM). Treatment of AK with imiquimod (IMIQ) 5\% cream has been shown to 'highlight' subclinical lesions. Objective: The aim of this study was to test the applicability of RCM for noninvasive monitoring of actinic field cancerization and detection of subclinical AK. Subjects and Methods: AK and surrounding skin sites with no apparent AK of 11 volunteers were selected for imaging and subsequently classified as 'clinical' and 'subclinical' AK. IMIQ was used 3 times weekly for 4 weeks. Results: RCM was able to detect morphologic features of AK in both clinical and subclinical AK; features were more pronounced in clinical lesions. The immunomodulatory response induced by IMIQ was visualized by RCM. Conclusion: Our findings indicate that RCM allows noninvasive monitoring of treatment response in vivo and permits early detection of subclinical AK, thus substantiating the incentive for therapy.
\end{abstract}

Copyright $\odot 2009$ S. Karger AG, Basel

\section{KARGER}

Fax +4161306 1234 E-Mail karger@karger.ch www.karger.com
(C) 2009 S. Karger AG, Basel

$1018-8665 / 10 / 2201-0015 \$ 26.00 / 0$

Accessible online at:

www.karger.com/drm

\section{Introduction}

Actinic keratoses (AK) are the most common malignant skin neoplasms and have been histopathologically classified as cutaneous squamous cell carcinoma in situ $[1,2]$. Sites of predilection are areas of chronic, high-dose or intermittent sun exposure, favoring the face, neck and forearms in persons with Fitzpatrick skin phototype IIII. As a ubiquitous carcinogen, UV radiation induces the initiation and promotion of progressive keratinocyte dysplasia, often affecting large areas of skin [3]. This etiopathogenetic concept has been referred to as 'actinic field cancerization'; AK represents one stage in the continuum from subclinical keratinocyte dysplasia to invasive squamous cell carcinoma $[4,5]$. The diagnosis of AK is generally based on clinical examination. However, biopsy and histological evaluation are performed in clinically indistinct cases and if invasive squamous cell carcinoma is suspected. While visual inspection may fail to detect early subclinical changes, invasive, repeated histological analysis of areas with extensive actinic damage may often not be feasible.

In the past decade, a number of novel treatment modalities have been developed and evaluated regarding their efficacy and safety. Imiquimod (IMIQ) 5\% cream 
represents the first topical immunomodulator approved for the treatment of genital warts, superficial basal cell carcinoma and AK [6-8]. For the treatment of AK, complete response rates ranging from 50 to $84 \%$ have been reported [7, 9]. Recently, new insights have been gained concerning the mode of action of IMIQ $5 \%$ cream. It acts as an agonist of toll-like receptors 7 and 8 resulting in activation of NF- $\kappa B$ and subsequent induction of proinflammatory cytokines including among others INF- $\alpha$, TNF- $\alpha$, IL-2, IL-6, IL- 8 and IL-12 [10]. In this induction, a central role has been ascribed to antigen-presenting dendritic cells, responding to low threshold concentrations of IMIQ $[11,12]$. Following topical application of IMIQ, the maturation and migration of epidermal Langerhans cells to regional lymph nodes has been demonstrated in vivo [10]. Summarizing current observations, the mode of action is based on the activation of cytotoxic $T$ cells with antitumoral and proapoptotic properties and induction of the innate immune response, which - in an independent pathway via the activation of the caspase cascade - results in mitochondrial-mediated apoptosis $[13,14]$. A number of clinical trials and observational analyses have shown the efficacy of IMIQ treatment for AK. While it has been demonstrated that IMIQ is able to induce a prominent inflammatory response in areas of visible AK, selected subclinical lesions may show a comparable response [15]. In the context of actinic field cancerization, this has also been referred to as the visualization of 'subclinical lesions'. Similarly, some studies reported a cosmetic improvement of areas with actinic photodamage following treatment with IMIQ, suggesting an overall regenerative effect of this immunomodulatory treatment $[16,17]$.

Reflectance confocal microscopy (RCM) is a noninvasive imaging technique that allows the visualization of cellular and subcellular structures of the skin in vivo with near histological resolution. In contrast to histological evaluation, which visualizes vertical sections of the tissues, RCM obtains horizontal (en face) optical sections in gray scale. A digital camera (Viva Cam, Lucid Inc., Rochester, N.Y., USA) connected to the RCM computer obtains dermoscopic images which may then be directly correlated to RCM evaluation and guide the identification of suspicious areas within the lesion [18]. A single image allows in vivo evaluation of a $500 \times 500 \mu \mathrm{m}$ area and by scanning of the microscope composite images, up to $8 \times 8 \mathrm{~mm}$ can be obtained. RCM has been used for the evaluation of a variety of inflammatory and neoplastic skin disorders [19-24]; recently, the clinically applicability of this method for the evaluation of AK has been in- vestigated [25-27]. It was postulated that initial changes of epidermal morphology and cellular atypia may be visualized by RCM before becoming clinically apparent. Therefore RCM may be useful for the evaluation of actinic field cancerization and the detection of subclinical AK. Moreover, the lesions may be examined in vivo and repeatedly over time, enabling a noninvasive analysis of treatment effects by RCM.

Based on these hypotheses, the primary goal of this study was to evaluate and describe the inflammatory and regenerative pharmacodynamic changes induced by IMIQ treatment over time. The secondary aim of this study was to test the possibility of detecting subclinical AK using RCM by correlating the microscopic observations to the clinically visible immunologic response to IMIQ.

\section{Subjects and Methods}

\section{Participants}

11 otherwise healthy Caucasian volunteers (Fitzpatrick skin phototype II-III), aged between 59 and 77 years, with a clinical diagnosis of AK of the face and scalp and signs of field cancerization were recruited to participate in this study. Subjects with a history of significant other skin disease were excluded from the protocol. Clinical investigations were conducted according to the principles of the Declaration of Helsinki and consent was obtained prior to enrollment. All 11 subjects completed the study and data of all subjects were included in the analysis.

\section{Clinical Evaluation}

All participants were examined for the presence of AK following the guidelines of the visual inspection and diagnosis of skin cancer [28]. 11 skin sites clinically suspicious for AK (subsequently defined as clinical AK) and 11 perilesional, clinically uninvolved skin sites (subsequently defined as subclinical AK) were selected for RCM analysis. Clinical photographs of all skin sites were obtained using a digital camera (Sony Cybershot 7.2, Sony Corp., Tokyo, Japan; Canon 350, Canon, Tokyo, Japan) under standardized room and lighting conditions. Transparency body charts were used to outline the selected skin areas at baseline to permit colocalization during follow-up examination for the duration of the study. Similarly, highlighting of new skin areas was documented on the chart by using permanent marker pens in a color-coded fashion, correlating individual colors to selected evaluation dates.

\section{Treatment Protocol}

IMIQ (MEDA Pharma, Sweden) was applied to selected study sites at recommended intervals 3 times weekly for a total of 4 weeks. Patients were requested to document their application regimen and possible treatment-related side effects using a patient diary. 
Table 1. Presence of selected features in clinical and subclinical AK sites

Subclinical Clinical AK AK

\section{Main features}

Parakeratosis/hyperkeratosis

Stratum corneum disruption

Individual corneocytes

SG - pleomorphism

SG - architectural disruption

SS - pleomorphism

SS - architectural disruption

Additional features

Solar elastosis

Increased vascularity/blood vessel dilatation $\bigcirc$

$\mathrm{SG}=$ Stratum granulosum; $\mathrm{SS}=$ stratum spinosum

$\bigcirc=$ Absent; $\bullet=$ present .

\section{In vivo RCM Evaluation}

A commercially available in vivo RCM device (Vivascope 1500, MAVIG GmbH, Munich, Lucid-Tech Inc., Henrietta, N.Y., USA) was used for imaging of all test sites. A detailed description of this technique and the device has been published [29-31]. Each skin site was systematically evaluated by RCM: one X-Y horizontal mapping (map dimensions: $4 \mathrm{~mm} \times 4 \mathrm{~mm}$ ) was performed at each epidermal layer beginning with the stratum corneum through the entire epidermis and into the upper reticular dermis. Similarly, 4-6 individual RCM images were captured in each epidermal layer, following a standardized evaluation protocol and individually subjected to descriptive analysis during the evaluation process. Based on previously defined RCM criteria, specific study sites were evaluated for the presence or absence of RCM features of AK [25-27]. The presence of main features was indicative of AK: disruption of the stratum corneum, individual corneocytes, parakeratosis/hyperkeratosis, overall architectural disruption, nuclear atypia/cellular pleomorphism at the level of the basal, spinous and granular layer. Additional features were used as follows: spongiosis, exocytosis, solar elastosis, increased vascularity/blood vessel dilatation, lymphocyte rolling, superficial inflammatory infiltrate. Based on these criteria, the presence of nuclear atypia/cellular pleomorphism was mandatory for a diagnosis of AK using RCM. The investigator (M.U.) performed the evaluation of all test sites and was not blinded to the clinical diagnosis before initiating RCM evaluation.

Detection of Clinical/Subclinical AK. Areas that showed main features of AK on RCM evaluation in the presence of clinical findings were subsequently defined as 'clinical AK'; corresponding areas that showed main features of AK on RCM evaluation in the absence of clinical findings were defined as 'subclinical AK'.

Therapeutic Monitoring. To detect the morphologic changes in study sites following topical applications of IMIQ 5\% cream, serial RCM evaluations were performed at selected intervals, including baseline (before treatment), 2 weeks after initiation of therapy and 4 weeks after end of treatment. The presence or absence of RCM features of AK was recorded as described above (table 1); similarly features of inflammatory response, including the presence of spongiosis, inflammatory cells, intraepidermal necrosis and superficial impetiginization or crusting, were documented. Clinical images illustrating the course of IMIQ treatment are shown in figure 7.

\section{Results}

\section{Clinical AK}

\section{RCM Evaluation of Clinical AK before Initiation of} Treatment (Baseline)

At the level of the stratum corneum, single detached keratinocytes seen as bright polygonal structures and clinically corresponding to hyperkeratotic scaling were visualized in all evaluation sites. At the level of the stratum spinosum and granulosum, atypical keratinocytes with cellular and nuclear pleomorphism were seen. An overall disarray of epidermal architecture with loss of the normal honeycomb pattern was visualized (11/11). At the level of the superficial dermis, blood vessel dilatation $(8 / 11)$ and solar elastosis (11/11) were seen (fig. 1).

\section{RCM Evaluation of Clinical AK after 2 Weeks of IMIQ Treatment}

Upon clinical evaluation, a notable inflammatory response was documented in 10/11 patients. One patient (patient 1) did not show any local or systemic response but continued treatment and was followed by RCM.

RCM evaluation of AK during treatment with IMIQ showed persistent superficial disruption at the level of the stratum corneum. At the level of the stratum granulosum and spinosum, persisting cellular and nuclear atypia was seen. At the level of the dermis, bright irregular bundles were visualized, representing solar elastosis. As aspects of inflammation/immunmodulation, RCM evaluation at the level of the stratum corneum revealed aggregates of small, weakly refractive cells clinically corresponding to superficial impetiginization/crusting in $7 / 11$ patients (not shown). In these areas, optical penetration depth was limited, partially interfering with resolution of deeper layers, impeding the visualization of cellular detail and inflammatory response in $4 / 11$ patients. RCM evaluation at the level of the epidermis and dermis revealed large, dendritic structures of bright appearance as well as small round bright cells of $10 \mu \mathrm{m}$ in diameter scattered among keratinocytes (6/11). Dendritic cells of two morphological subtypes were visualized by RCM: one of spindle 
Fig. 1. Representative RCM images of clinical $\mathrm{AK}$ before initiation of treatment. a RCM image obtained at the level of the stratum corneum illustrating superficial disruption with large polygonal structures representing single detached keratinocytes (arrows). b, c RCM image obtained at the level of the stratum granulosum (b) and stratum spinosum (c) showing cellular and nuclear atypia with variation in cell size and form resulting in an atypical honeycomb pattern. The structure in the lower right corner represents a hair follicle (HF). d RCM image taken at the level of the upper reticular dermis showing bright irregular bundles and lace-like amorphous material representing solar elastosis. Small canalicular spaces can be seen representing dilated blood vessels.
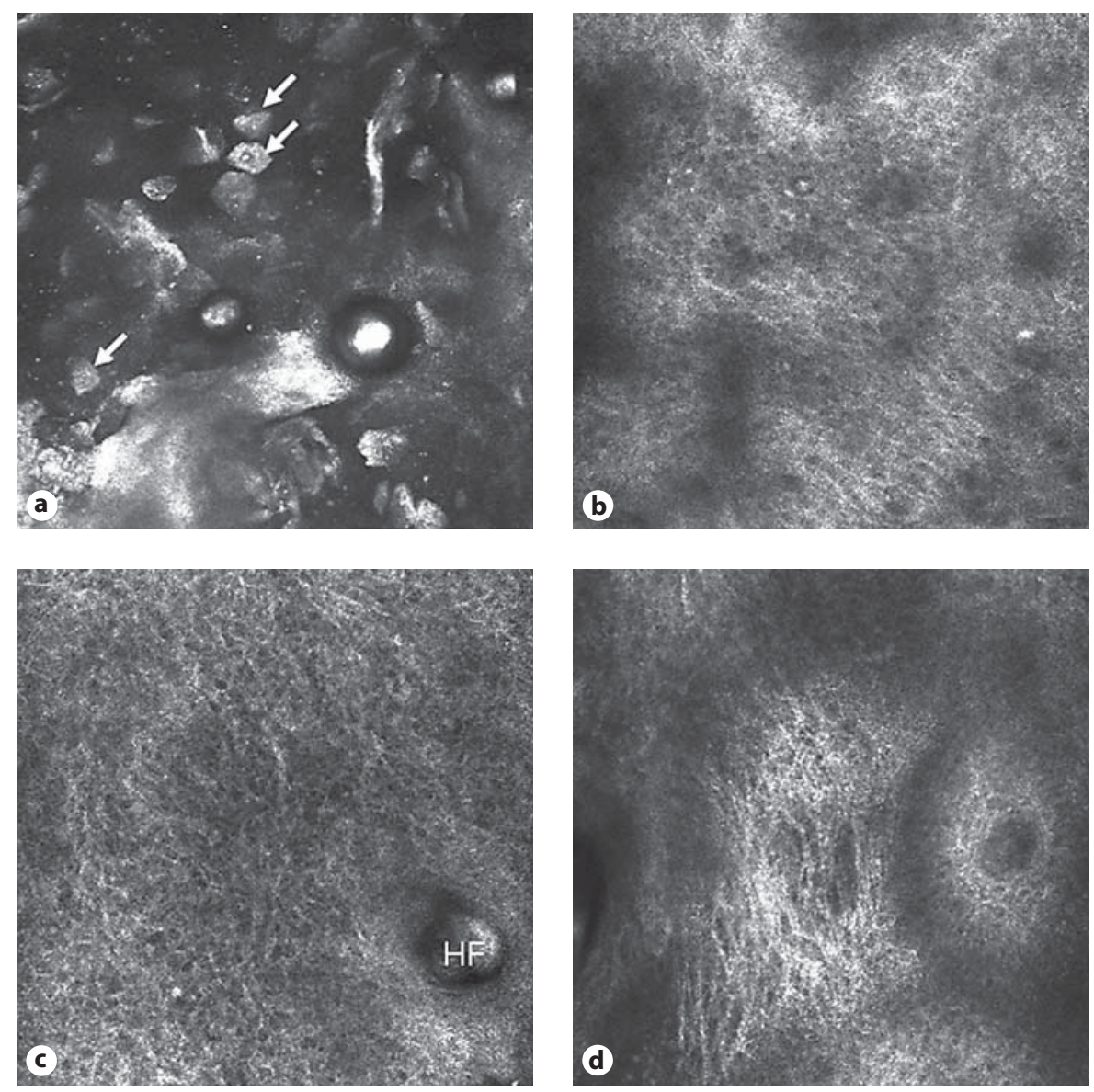

shape and high reflectance, resembling melanocytes as described by Pellacani et al. [21] and the other of large shape and bright appearance, most likely corresponding to epidermal (Langerhans) or dermal dendritic cells or melanophages. At the level of the dermis, the presence of small round bright cells was visualized in a perifollicular and perivascular distribution (6/11). Blood vessels of increased diameter were seen and high blood flow was noted on in vivo examination (6/11) (fig. 2).

RCM Evaluation of Clinical AK 4 Weeks after the End of IMIQ Treatment

Clinical examination showed clearance of AK in 9/11 patients; patient 1 did not to show an identifiable treatment response on clinical examination and was thus classified as a nonresponder. Another patient (patient 2) showed clearance on clinical examination but persistence of AK features (atypia of the stratum granulosum and spinosum) and inflammation on RCM examination. Overall, the stratum corneum showed a more homoge- neous appearance and increased cohesiveness of corneocytes comparable to findings observed in normal, unaffected skin sites [25-27]. RCM images obtained at the level of the stratum granulosum and spinosum showed a more regular appearance of keratinocytes, with polygonal cells arranged in a typical honeycomb pattern (9/11). At the level of the dermis, the persistence of bright, irregularly configurated bundles with lace-like amorphous material suggestive of solar elastosis was seen in all patients. Superficial dermal blood vessels showed a regular appearance in the majority of patients (8/11) with no residual inflammatory infiltrate present (fig. 3).

\section{Subclinical AK}

\section{RCM Evaluation of Subclinical AK before Initiation of Treatment (Baseline)}

At the level of the stratum corneum, a cohesive layer of corneocytes with homogeneous bright reflectance and 
Fig. 2. RCM morphology of clinical AK 2 weeks after initiation of IMIQ treatment. a RCM image obtained at the stratum spinosum illustrating atypical keratinocytes and the presence of large, spindle-shaped dendritic cells (dashed circle) and small, highly refractive structures (arrows). b RCM image obtained at the suprabasal layer showing increased brightness of suprabasal keratinocytes. The cells measure around $20 \mu \mathrm{m}$ in diameter and have large dark nuclei and bright cytoplasm (dashed circles). Furthermore, small round bright cells of around $10 \mu \mathrm{m}$ can be identified, which likely correspond to inflammatory cells (arrows). c RCM image obtained at the level of the dermo-epidermal junction showing 3 large very bright cells of dendritic appearance in perifollicular distribution (dashed circles), surrounded by several round bright cells suggestive of inflammatory cells. d RCM image obtained at the level of the papillary dermis with presence of prominent blood vessels (BV) that appear as bright canalicular structures and showed increased blood flow on in vivo examination. Furthermore, a perivasular and perifollicular inflammatory infiltrate consisting of small round bright cells can be seen.
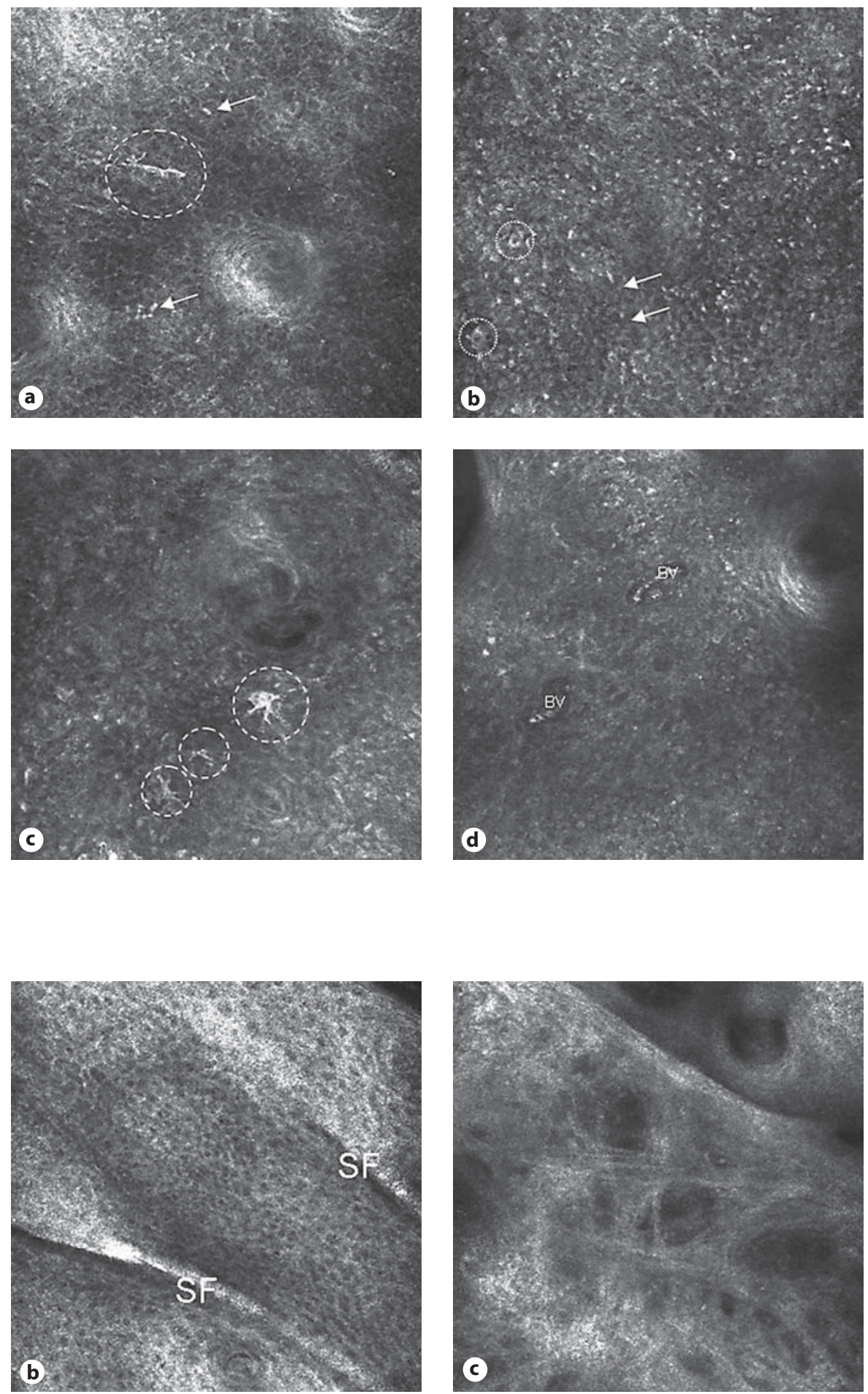

Fig. 3. RCM morphology of clinical AK 4 weeks after the end of treatment. a RCM image obtained at the level of the stratum corneum showing homogeneous appearance and coherence of corneocytes. b RCM image obtained at the level of the stratum spinosum with regular keratinocytes arranged in a typical honey- comb pattern and visualization of typical skin folds (SF). c RCM image obtained at the level of the upper reticular dermis with thickened collagen bundles and moderately refractile lace-like amorphous material illustrating remaining solar elastosis. 
Fig. 4. Representative RCM images of subclinical AK before initiation of treatment. a RCM image obtained at the level of the stratum corneum, which appears homogeneously bright with a cohesive aspect. Highly refractive islands are visible in between skin folds (SF). b RCM image obtained at the level of the stratum granulosum. Nuclei of granular keratinocytes appear as homogeneous monomorphous dark polygonal structures forming a characteristic honeycomb pattern. c RCM images obtained at the level of the stratum spinosum. Cellular and nuclear atypia can be seen that resulted in the disruption of the epidermal architecture with focal loss of the honeycomb pattern (dashed circle). Arrows indicate nuclear polymorphism. d RCM image obtained at the level of the reticular dermis illustrating solar elastosis with irregular bundles of bright reflectance and moderately refractile lace-like structures.
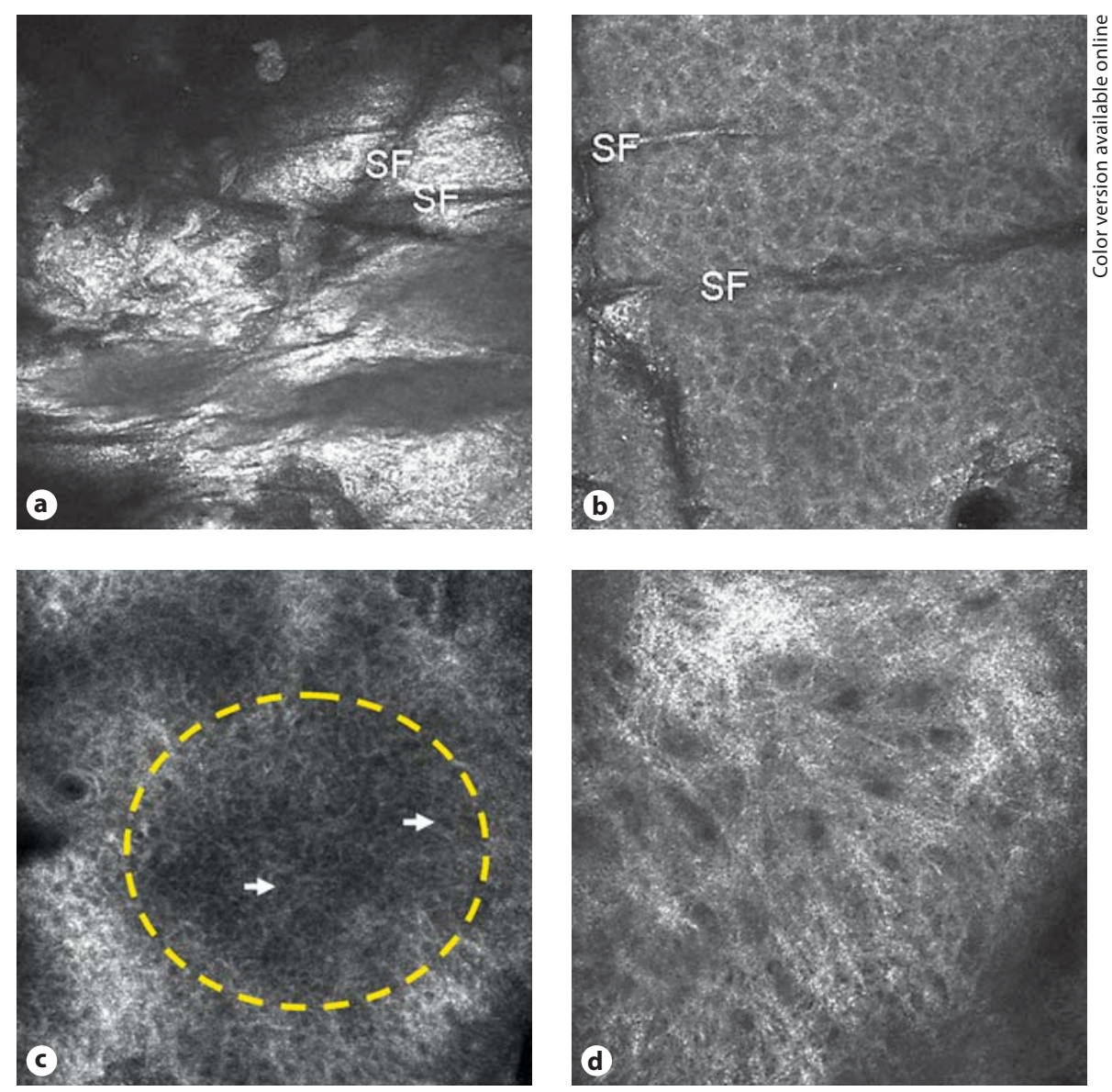

a regular appearance was observed in 9/11 patients. 2/11 patients showed slight superficial stratum corneum disruption with single detached corneocytes. The stratum granulosum showed polygonal, uniform cells arranged in a typical honeycomb pattern in the majority of patients $(9 / 11)$. At the level of the stratum spinosum, discrete cellular and nuclear atypia of the keratinocytes was visualized, resulting in focal disruption of the epidermal architecture (11/11). At the level of the dermis, bright irregular bundles were visualized, suggestive of solar elastosis (11/11). Furthermore, small canalicular bright structures morphologically corresponding to dilated blood vessels were seen in $3 / 11$ patients (fig. 4 ).

\section{RCM Evaluation of Subclinical AK 2 Weeks after \\ Initiation of IMIQ Treatment}

Clinical evaluation revealed a notable inflammatory response in $10 / 11$ patients. Patient 1 did not show any signs of clinical response.
RCM evaluation of subclinical sites showed persistence of RCM features of AK in all lesions at the level of the basal and spinous layer with atypia of keratinocytes and nuclear pleomorphism. In contrast to the baseline evaluation, superficial disruption and impetiginization/ crusting was seen, partially interfering with optical penetration and impeding the visualization of inflammatory response in 2/11 lesions. Variable degrees of inflammation were detected within the epidermis and dermis, seen as small bright round cells and large dendritic cells in $8 / 11$ patients. The presence of single denticulated large cells may likely correspond to apoptotic keratinocytes. At the level of the suprabasal layer cells two characteristic cell types were frequently observed: one larger type of approximately $15 \mu \mathrm{m}$ in diameter and a dark nucleus surrounded by a highly refractive cytoplasm consistent with pigmented basal cells; scattered small bright cells of approximately $10 \mu \mathrm{m}$ corresponding to inflammatory infiltrate were visualized. Similarly, numerous small bright round cells were detected around hair follicles and were 
Fig. 5. RCM morphology of subclinical AK 2 weeks after initiation of IMIQ treatment. a RCM image obtained at the border of stratum granulosum and stratum spinosum showing a prominent perifollicular epidermal infiltrate with numerous bright round cells scattered among spinous keratinocytes (arrowheads) and marked disruption of the epidermal architecture. Single denticulated large cells can be seen, possibly corresponding to apoptotic keratinocytes (dashed circle). b RCM image obtained at the level of the suprabasal layer showing cells with dark nucleus and bright cytoplasm corresponding to pigmented basal cells (dashed circles) and scattered small bright cells corresponding to an inflammatory infiltrate (arrowheads). c RCM image obtained at the level of the papillary dermis showing numerous small round cells with a pronounced bright appearance perifollicularly and scattered among collagen bundles (arrowheads). d RCM image obtained at the level of the upper reticular dermis illustrating large canalicular spaces filled with small round cells of medium reflectance which correspond to dilated bloods vessels. On in vivo examination, increased blood flow was seen.
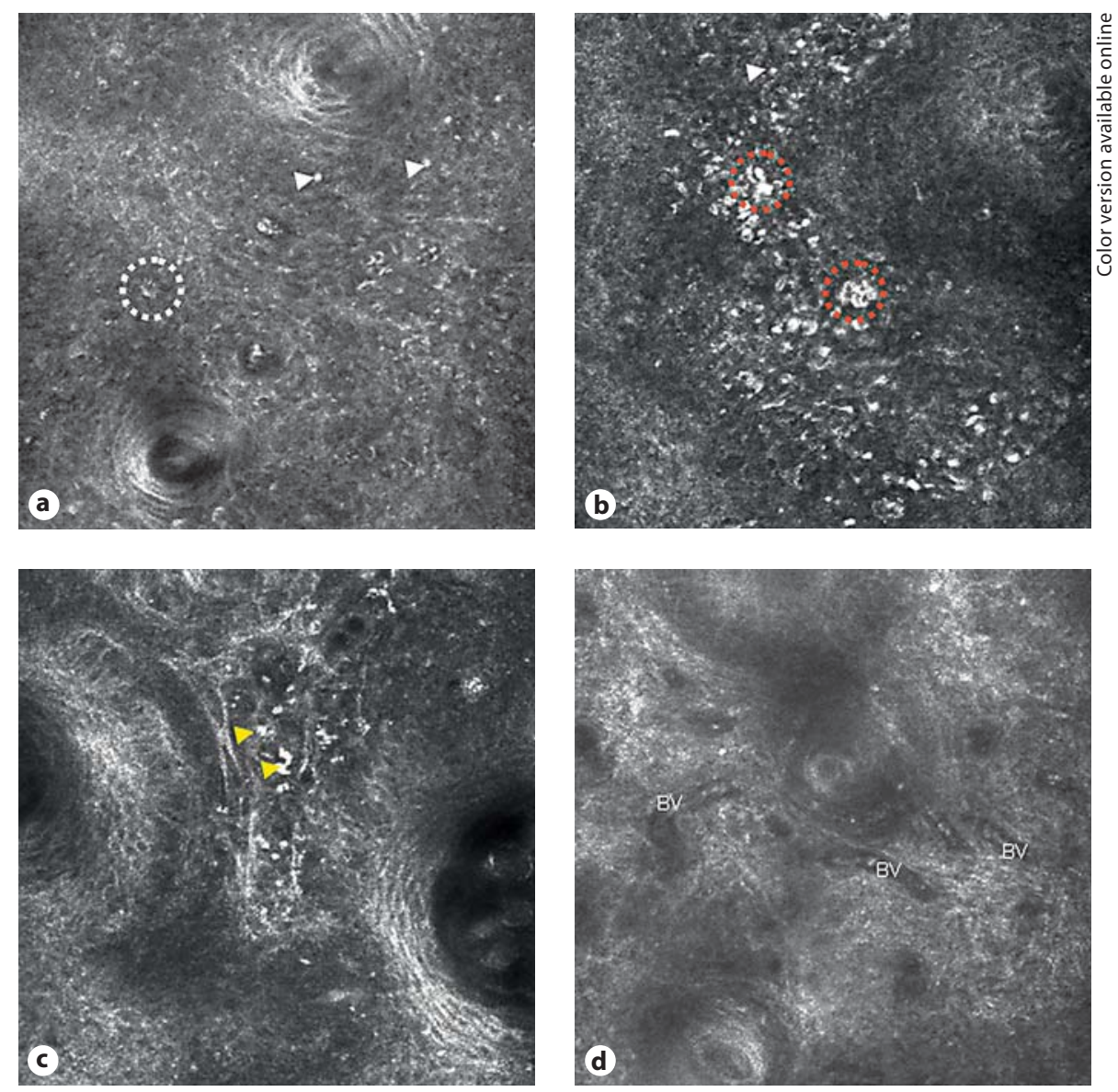

interspersed in between collagen bundles. Large canalicular spaces filled with small round cells of medium reflectance and increased flow on in vivo examination were visualized that correspond to dilated and elongated blood vessels (8/11) (fig. 5).

\section{RCM Evaluation of Subclinical AK 4 Weeks after the End of IMIQ Treatment}

Upon clinical evaluation, resolution of inflammation was noted in all patients with a previously documented treatment response. The nonresponder (patient 1) did not show significant changes when compared to the baseline visit in both clinical and RCM evaluation. Patient 2 showed no clinical findings of AK while RCM features of $\mathrm{AK}$ and inflammation persisted. The stratum corneum appeared homogeneously bright and showed cohesion of tight corneocytes in 10/11 patients. At the level of the stratum granulosum and spinosum, polygonal cells of regular appearance and homogeneous arrangement were seen in the majority of patients (8/11), resulting in a typical honeycomb pattern. Interpretation of RCM data was limited in 1 patient due to poor image quality. At the level of the dermis, persistence of bright thickened bundles was observed (fig. 6).

\section{Discussion}

The incidence of $\mathrm{AK}$ has been consistently rising, now accounting for the second most common cause for dermatology consultations in the United States [32]. AK typically arise in areas of chronic sun exposure of the face, neck and forearms and represent one stage in the continuum from subclinical keratinocyte dysplasia to invasive squamous cell carcinoma. The diagnosis of $\mathrm{AK}$ is generally based on clinical inspection, and studies have shown positive predictive values for clinical diagnosis between 81 and 94\% when compared to histopathologic examination $[33,34]$. Biopsy with histological evaluation is not routinely performed in the diagnostic work-up for 

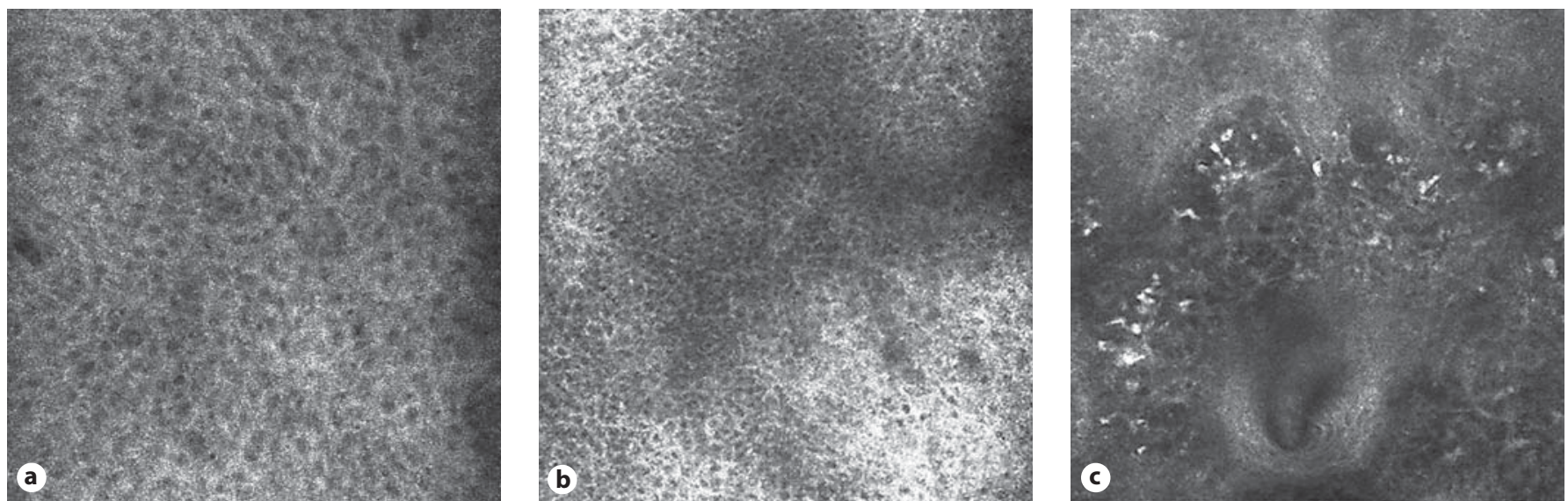

Fig. 6. RCM morphology of subclinical AK 4 weeks after the end of treatment. a RCM image obtained at the level of the stratum granulosum showing granular keratinocytes homogeneously arranged in a typical honeycomb pattern. b RCM image obtained at the level of the stratum spinosum illustrating a slight architec- tural disarray with cellular and nuclear atypia and a disrupted honeycomb pattern. c RCM image obtained at the level of the papillary dermis showing persistence of small, highly refractive round cells in between collagen bundles.
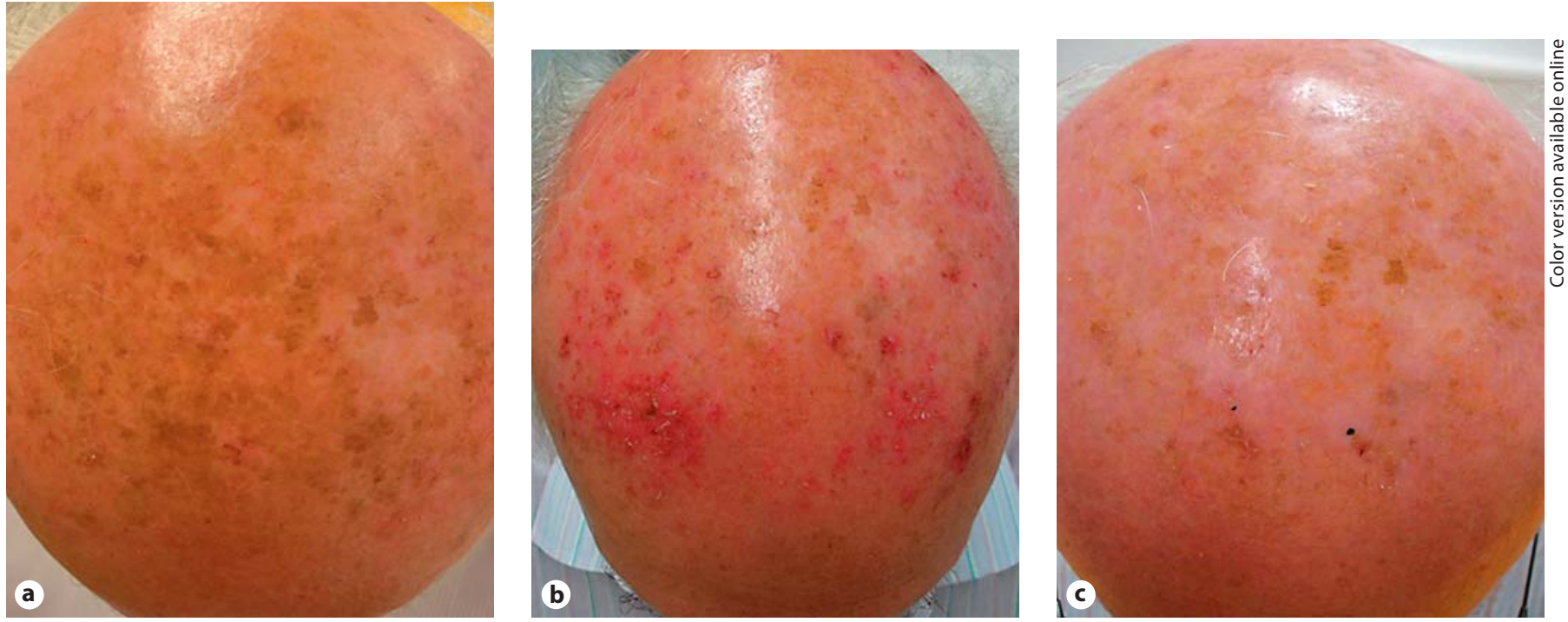

Fig. 7. Clinical images illustrating the clinical course IMIQ treatment for field cancerization on the scalp. a Clinical images illustrating actinic field cancerization before initiation of IMIQ treatment. b Clinical photograph illustrating inflammatory response after 2 weeks of IMIQ application with erythema and mild erosions and crusting. c Clinical images obtained 4 weeks after the end of treatment illustrating remission of inflammatory response and normal-appearing skin without sign for AKs.
AK, nor is histology generally performed to evaluate treatment efficacy, with the exception of selected outcome studies and for the detection of residual, recurrent or invasive disease. In an attempt to increase diagnostic accuracy with noninvasive imaging technologies, a number of adjunct diagnostic devices have been evaluated for their clinical applicability. Among others, recent studies reported high sensitivity and specificity rates for diagnosis of AK by RCM in vivo [26, 27].

Similarly, the access to noninvasive, topical treatment modalities has greatly facilitated the management of AK [35]. Among them the topical immunomodulator IMIQ 
is now widely used in dermatologic therapy. Its mode of action implies the induction of the innate immunity resulting in a selective antitumoral immune response through the activation of dendritic cells, cytotoxic T lymphocytes and proinflammatory cytokines. RCM has been used for monitoring and evaluation of treatment efficacy of basal cell carcinoma and lentigo maligna melanoma during topical IMIQ therapy [36-38]. To our knowledge, no systematic RCM evaluation of treatment response of AK to IMIQ has been performed.

The primary objective of this study was to evaluate and describe the dynamic morphologic changes induced by IMIQ treatment over time. Furthermore we aimed to test the possibility of detecting subclinical AK using RCM in order to confirm this hypothesis by the clinically visible response to IMIQ.

Following baseline evaluations of clinical and subclinical AK, all patients participating in this study were asked to apply IMIQ 5\% cream in a defined study area 3 times weekly for a total of 4 weeks, following established dosing schemes. Neoplastic, inflammatory, regenerative and vascular changes were documented in both clinical and subclinical AK sites using RCM evaluation parameters for $\mathrm{AK}$ as published previously [26, 27].

RCM was able to identify subclinical AK by visualization of cellular and nuclear atypia within the spinous cell layer. After 2 weeks of treatment, RCM morphologic features of inflammatory response were detected by RCM in vivo and included the presence of small bright cells and two types of dendritic cells scattered among keratinocytes and the superficial dermis. The spindle-shaped, bright dendritic cells most likely correspond to melanocytes [39], whereas the plumb bright dendritic cells may correspond to Langerhans or dermal dendritic cells, which have previously been shown histologically to belong to the inflammatory infiltrate in IMIQ-treated lesions [40]. The presence of the melanocytes may be explained by the chronically sun-damaged skin with mottled hyperpigmentation that is often associated with AK. Furthermore, the detection of denticulated large cells within the epidermis may correspond to apoptotic keratinocytes, an observation that has previously been described in the mouse model [10].

Furthermore, RCM was able to detect residual atypia in 2 patients after end of treatment without any clinical aspects of AK. These findings were suggestive of incomplete clearance of $\mathrm{AK}$, subsequently prompting a second cycle of IMIQ therapy. In that context, it may be noted that following noninvasive therapy, a histopathologic control of clearance is not routinely performed and not feasible. By using RCM for evaluation of treatment efficacy, the early, noninvasive detection of residual atypia may ultimately decrease recurrence rates.

Limitations of the technique include the time needed for image acquisition in large anatomic areas as well as inherent optical limitations interfering with resolution of cellular details. The optical resolution and penetration is especially limited in hyperkeratotis lesions such as squamous cell carcinoma, verrucous lesions or hyperkeratotic $\mathrm{AK}$ and may interfere with image quality and correct diagnosis may often not be obtained by RCM. While the technique is easily learned, the results remain strongly user-dependent, and reproducibility of results may vary between investigators.

In conclusion, our findings indicate that the use of RCM allows the noninvasive visualization and monitoring of sequential pharmacodynamic changes during IMIQ therapy over time. Thereby, RCM may expand our insights into the mode of action of this immunmodulatory treatment and facilitate the evaluation of treatment efficacy. Furthermore, RCM may aid in the detection of subclinical AK in the setting of field cancerization, potentially increasing diagnostic accuracy compared to clinical evaluation alone. The adjunct use of noninvasive optical techniques may allow the early identification of affected skin sites, substantiate the incentive for therapy and ultimately increase therapeutic efficacy.

\section{Conflict of Interest}

M. Ulrich was awarded a research grant by MEDA Pharma GmbH, Sweden; M. Ulrich, S. Astner and E. Stockfleth are lecturers for MAVIG GmbH, Germany; E. Stockfleth is a consultant for MEDA Pharma GmbH.

\footnotetext{
References

1 Heaphy MR Jr, Ackerman AB: The nature of actinic keratosis: a critical review in a historical perspective. J Am Acad Dermatol 2000;43:138-150

2 Ackerman AB: Solar keratosis is squamous cell carcinoma. Arch Dermatol 2003;139: 1216-1217.

-3 Callen JP, Bickers DR, Moy RL: Actinic keratoses. J Am Acad Dermatol 1998;39:134136.

-4 Braakhuis BJ, Tabor MP, Kummer JA, Leemans CR, Brakenhoff RH: A genetic explanation of Slaughter's concept of field cancerization: evidence and clinical implications. Cancer Res 2003;63:1727-1730.
} 
5 Slaughter DP, Southwick HW, Smejkal W: Field cancerization in oral stratified squamous epithelium; clinical implications of multicentric origin. Cancer 1953;6:963968.

-6 Edwards L, Ferenczy A, Eron L, Baker D, Owens ML, Fox TL, Hougham AJ, Schmitt KA: Self-administered topical 5\% imiquimod cream for external anogenital warts. HPV Study Group. Human Papillomavirus. Arch Dermatol 1998;134:25-30.

-7 Stockfleth E, Meyer T, Benninghoff B, Salasche S, Papadopoulos L, Ulrich C, Christophers E: A randomized, double-blind, vehicle-controlled study to assess $5 \%$ imiquimod cream for the treatment of multiple actinic keratoses. Arch Dermatol 2002;138: 1498-1502.

-8 Lebwohl M, Dinehart S, Whiting D, Lee PK, Tawfik N, Jorizzo J, Lee JH, Fox TL: Imiqui$\bmod 5 \%$ cream for the treatment of actinic keratosis: results from two phase III, randomized, double-blind, parallel group, vehicle-controlled trials. J Am Acad Dermatol 2004;50:714-721.

-9 Hadley G, Derry S, Moore RA: Imiquimod for actinic keratosis: systematic review and meta-analysis. J Invest Dermatol 2006;126: 1251-1255.

10 Schön MP, Schön M: Imiquimod: mode of action. Br J Dermatol 2007;157(suppl 2):813.

-11 Zhu KJ, Cen JP, Lou JX, Wang Q, Zhang X, Xu Y, Chen XZ, Cheng H: Imiquimod inhibits the differentiation but enhances the maturation of human monocyte-derived dendritic cells. Int Immunopharmacol 2009;9: 412-417.

12 Burns RP Jr, Ferbel B, Tomai M, Miller R, Gaspari AA: The imidazoquinolines, imiquimod and R-848, induce functional, but not phenotypic, maturation of human epidermal Langerhans' cells. Clin Immunol 2000;94:13-23.

13 Meyer T, Nindl I, Schmook T, Ulrich C, Sterry W, Stockfleth E: Induction of apoptosis by toll-like receptor-7 agonist in tissue cultures. Br J Dermatol 2003;149(suppl 66):9-14.

14 Schön MP, Schön M: Immune modulation and apoptosis induction: two sides of the antitumoral activity of imiquimod. Apoptosis 2004;9:291-298.

15 Del Rosso JQ: The use of topical imiquimod for the treatment of actinic keratosis: a status report. Cutis 2005;76:241-248.

16 MetcalfS, Crowson AN, Naylor M, Haque R, Cornelison R: Imiquimod as an antiaging agent. J Am Acad Dermatol 2007;56:422425 .
17 Smith K, Hamza S, Germain M, Skelton H: Does imiquimod histologically rejuvenate ultraviolet radiation-damaged skin? Dermatol Surg 2007;33:1419-1428; discussion 1428-1429.

18 Scope A, Ardigo M, Marghoob AA: Correlation of dermoscopic globule-like structures of dermatofibroma using reflectance confocal microscopy. Dermatology 2008;216:8182.

19 Nori S, Rius-Diaz F, Cuevas J, Goldgeier M, Jaen P, Torres A, González S: Sensitivity and specificity of reflectance mode confocal microscopy for in-vivo diagnosis of basal cell carcinoma: a multicenter study. J Am Acad Dermatol 2004;51:923-930.

20 Aghassi D, Anderson RR, Gonzalez S: Confocal laser microscopic imaging of actinic keratoses in vivo: a preliminary report. J Am Acad Dermatol 2000;43:42-48.

21 Pellacani G, Guitera P, Longo C, Avramidis $\mathrm{M}$, Seidenari S, Menzies S: The impact of in vivo reflectance confocal microscopy for the diagnostic accuracy of melanoma and equivocal melanocytic lesions. J Invest Dermatol 2007;127:2759-2765.

-22 Ardigo M, Cota C, Berardesca E, González S Concordance between in vivo reflectance confocal microscopy and histology in the evaluation of plaque psoriasis. J Eur Acad Dermatol Venereol 2009;23:660-667.

23 Astner S, González S, Gonzalez E: Noninvasive evaluation of allergic and irritant contact dermatitis by in vivo reflectance confocal microscopy. Dermatitis 2006;17: 182-191.

24 Langley RG, Walsh N, Sutherland AE, et al: The diagnostic accuracy of in vivo confocal scanning laser microscopy compared to dermoscopy of benign and malignant melanocytic lesions: a prospective study. Dermatology 2007;215:365-372.

25 Aghassi D, Anderson RR, González S: Confocal laser microscopic imaging of actinic keratoses in vivo: a preliminary report. J Am Acad Dermatol 2000;43(1 pt 1):42-48.

26 Ulrich M, Maltusch A, Rius-Diaz F, RöwertHuber J, González S, Sterry W, Stockfleth E, Astner S: Clinical applicability of in vivo reflectance confocal microscopy for the diagnosis of actinic keratoses. Dermatol Surg 2008:34:610-619.

27 Horn M, Gerger A, Ahlgrimm-Siess V, Weger W, Koller S, Kerl H, Samonigg H, Smolle J, Hofmann-Wellenhof R: Discrimination of actinic keratoses from normal skin with reflectance mode confocal microscopy. Dermatol Surg 2008;34:620-625.

28 Guidelines for the management of actinic keratoses of the European Dermatology Forum. http://www.euroderm.org/content/ guidelines_keratoses.
29 Rajadhyaksha M, Grossman M, Esterowitz D, Webb RH, Anderson RR: In vivo confocal scanning laser microscopy of human skin: melanin provides strong contrast. J Invest Dermatol 1995;104:946-952.

30 Rajadhyaksha M, Anderson RR, Webb RH: Video-rate confocal scanning laser microscope for imaging human tissues in vivo. Appl Opt 1999;38:1-12.

- 31 Rajadhyaksha M, González S, Zavislan JM, Anderson RR, Webb RH: In vivo confocal scanning laser microscopy of human skin. II. Advances in instrumentation and comparison with histology. J Invest Dermatol 1999; 13:293-303

- 32 Spencer JM, Hazan C, Hsiung SH, Robins P: Therapeutic decision making in the therapy of actinic keratoses. J Drugs Dermatol 2005; 4:296-301.

-33 Venna SS, Lee D, Stadecker MJ, Rogers GS: Clinical recognition of actinic keratoses in a high-risk population. How good are we? Arch Dermatol 2005;141:507-509.

-34 Thompson SC, Jolley D, Marks R: Reduction of solar keratoses by regular sun-screen use. N Engl J Med 1993;329:1147-1151.

- 35 Stockfleth E, Ferrandiz C, Grob JJ, Leigh I, Pehamberger $\mathrm{H}$, Kerl $\mathrm{H}$; European Skin Academy: Development of a treatment algorithm for actinic keratoses: a European Consensus. Eur J Dermatol 2008;18:651-659.

-36 Torres A, Niemeyer A, Berkes B, Marra D, Schanbacher C, González S, Owens M, Morgan B: $5 \%$ imiquimod cream and reflectance-mode confocal microscopy as adjunct modalities to Mohs micrographic surgery for treatment of basal cell carcinoma. Dermatol Surg 2004;30(12 Pt 1):1462-1469.

37 Curiel-Lewandrowski C, Williams CM, Swindells KJ, Tahan SR, Astner S, Frankenthaler RA, González S: Use of in vivo confocal microscopy in malignant melanoma: an aid in diagnosis and assessemnt of surgical and nonsurgical therapeutic approaches. Arch Dermatol 2004;140:1127-1132.

-38 Goldgeier M, Fox CA, Zavislan JM, Harris D, González S: Noninvasive imaging, treatment and microscopic confirmation of clearance of basal cell carcinoma. Dermatol Surg 2003; 29:205-210.

39 Pellacani G, Cesinaro AM, Seidenari S: Reflectance-mode confocal microscopy for the in vivo characterization of pagetoid melanocytosis in melanomas and nevi. J Invest Dermatol 2005; 125:532-537.

40 Wolf IH, Kodama K, Cerroni L, Kerl H: Nature of inflammatory infiltrate in superficial cutaneous malignancies during topical imiquimod treatment. Am J Dermatopathol 2007;29:237-241 